\title{
Pain management in trauma patients in (pre)hospital based emergency care: current practice versus new guideline
}

\author{
AC Scholten MSc a,b; SAA Berben PhD, RN ${ }^{a, \$}$; AH Westmaas PhD ${ }^{a, c, d}$; PM van Grunsven e ; ET de Vaal, \\ MD f; PPM Rood, MD, PhD g; N Hoogerwerf, PhD, MD h,i; CJM Doggen PhD ${ }^{j}$; L Schoonhoven, PhD, RN \\ ${ }^{a, k, l}$; on behalf of the Emergency Pain Study Group
}

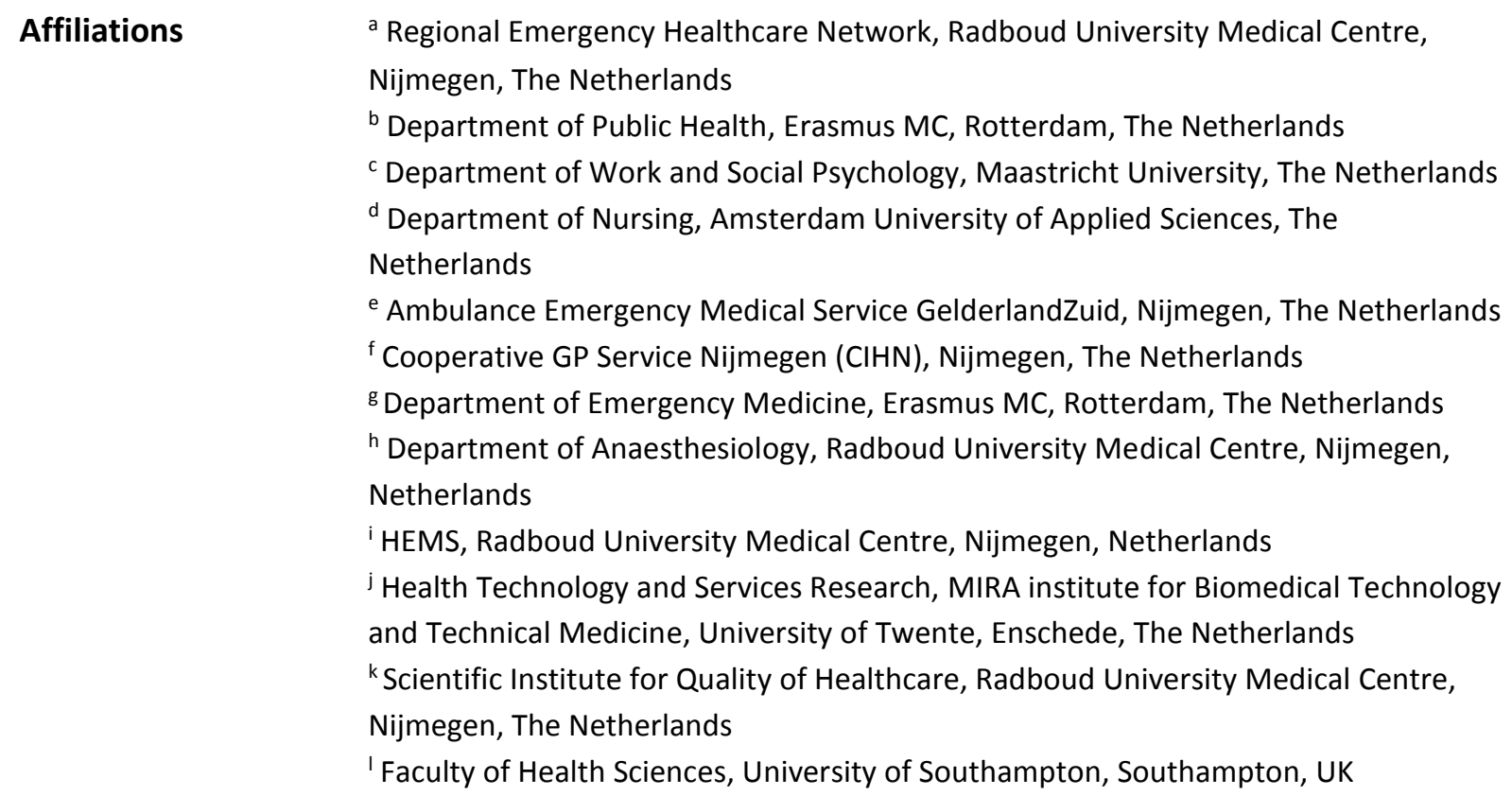

\section{\$Corresponding author}

Sivera A.A. Berben PhD

Radboud University Medical Centre

Internal Postal Code 911

Emergency Healthcare Network

PO Box 9101, 6500 HB Nijmegen, The Netherlands

Phone: +31247440080

E-mail: Sivera.Berben@radboudumc.nl

Keywords: Wounds and injuries, Trauma, Acute pain, Pain management, Guideline, Emergency care, Emergency Department, Emergency Medical Services 


\section{ABSTRACT}

Introduction Acute pain in trauma patients in emergency care is still undertreated. Early pain treatment is assumed to effectively reduce pain in patients and improve long-term outcomes. In order to improve pain management in the chain of emergency care, a national evidence-based guideline was developed. The aim of this study was to assess whether current practice is in compliance with the guideline 'Pain management for trauma patients in the chain of emergency care' from the Netherlands Association for Emergency Nurses (in Dutch NVSHV), and to evaluate early and initial pain management for adult trauma patients in emergency care.

Methods Chart reviews were conducted in three regions of the Netherlands using electronic patient files of trauma patients from the chain of emergency care. We included one after-hours General Practitioner Co-operation (GPC), one ambulance Emergency Medical Services (EMS), two Helicopter Emergency Medical Services (HEMS), and three Emergency Departments (EDs). Organisation of pain management, pain assessment, and pain treatment was examined and compared with national guideline recommendations, including quality indicators.

Results We assessed a random sample of 1,066 electronic patient files. The use of standardized tools to assess pain was registered in zero to $52 \%$ of the electronic patient files per organisation. Registration of (non-)pharmacological pain treatment was found in less than half of the files. According to the files, pharmacological pain treatment deviated from the guideline in $73 \%$ to $99 \%$ of the files. Time of administration of medication was missing in $73 \%$ to $100 \%$. Reassessment of pain following pain medication was recorded in half of the files by the HEMS, but not in files of the other organisations. 
Conclusions The (registration of) current pain management in trauma patients in the chain of emergency care varies widely between healthcare organisation, and deviates from national guideline recommendations. Although guideline compliance differs across groups of healthcare professionals, maximum compliance rate with indicators registered is $52 \%$. In order to improve pain management and evaluate its effectiveness, we recommend to improve pain registration in patient files. Furthermore, we advise to identify barriers and facilitators related to the implementation of the national guideline in all emergency care organisations.

\section{INTRODUCTION}

Treatment of acute pain in emergency care still gets insufficient attention. ${ }^{1}$ Acute pain and trauma are often closely related to one another, as pain is induced by noxious stimuli at the site of tissue damage. ${ }^{2}$ Recent studies show that the prevalence of pain in trauma patients in the Dutch (prehospital) emergency care setting is 70 to $91 \% \cdot{ }^{1,3}$ In contrast to the improved treatment of postoperative and chronic pain,,$^{4,5}$ the treatment of acute pain in emergency care is low; ${ }^{1}$ only 19 to $30 \%$ of trauma patients receive pharmacological pain treatment. ${ }^{1,3}$

Emergency care for trauma patients encompasses the care for patients with recent (within 24 hours) suspected injuries caused by blunt or penetrating forces, falls, explosions, heat/cold or chemical toxicants. In the Netherlands, emergency care is provided by General Practitioners (Co-operations) $(G P(C) s)$, ambulance Emergency Medical Services (EMS), Helicopter Emergency Medical Services (HEMS), and Emergency Departments (EDs). Collaboration between healthcare professionals in the chain of emergency care with respect to pain management is not optimal, making it very difficult to guarantee continuity of care. As a consequence, pain treatment is not always applied, not continued or contradicts the pain management by the preceding professional partner in the chain. ${ }^{6}$ The undertreatment of acute pain can have an adverse effect on the outcome of the treatment, e.g. a 
delay in wound healing, and causes a longer period of recovery. ${ }^{7}$ Furthermore, poorly treated acute pain can result in chronic pain. ${ }^{8}$ Early pain treatment is therefore of great importance.

To improve pain management in trauma patients, a national evidence-based guideline 'Pain management for trauma patients in the chain of emergency care' was developed in $2010 .{ }^{9}$ This multidisciplinary guideline from the Netherlands Association for Emergency Nurses (in Dutch NVSHV) provides clear recommendations and quality indicators for early pain management in evaluable, adult trauma patients in the chain of emergency care, concerning pain assessment, (non-) pharmacological pain treatment, and the organisation of pain management.

The objective of this study is to assess whether current practice is in compliance with the Dutch guideline recommendations, and to evaluate early and initial pain management for adult trauma patients in the chain of emergency care. The evaluation provides insight into the extent to which the guideline is already used by healthcare professionals in the chain of emergency care. It identifies the adherence and deviations in current practice from the quality indicators in the guideline, and provides a starting point for the implementation of the guideline.

\section{MATERIALS AND METHODS}

\section{Design}

Between January and March 2012 (HEMS from October 2011 to March 2012) chart reviews were conducted, assessing electronic patient files of trauma patients from seven organisations in the chain of emergency care, in three regions of the Netherlands; one after-hours GPC (suburban), one EMS (suburban), two HEMS (suburban and urban), and three EDs (rural/suburban, suburban and urban). Trauma patients were defined as patients with (suspected) injuries, due to mechanisms of blunt or penetrating forces, falls, explosions, heat/cold or chemical toxicants. ${ }^{10}$ Patient files were used to assess whether current practice is in compliance with the guideline 'Pain management for trauma 
patients in the chain of emergency care' from the NVSHV and to evaluate pain assessment and current pain management for adult trauma patients in emergency care.

\section{Setting}

The target group of the guideline consists of GPs, physicians and nurses in ambulance EMS and the ED, and team members of the HEMS. Dutch GPs organise their afterhours primary care - weekdays 5PM to 8AM and weekend - in large-scale GPCs. ${ }^{11}$ Telephone triage nurses assess the urgency of patient's health problem and decide - based on triage protocols ${ }^{12}$ and guidelines - the appropriate action to be taken, ${ }^{11}$ e.g. using paracetamol. In addition, out-of-hospital emergency care is also provided by nurses in EMS; registered nurses who followed a national training program and deliver pre-hospital care autonomously, based on national protocols. These national protocols assist the nurses in the limited use of Fentanyl, Ketanest (S-Ketamine), and Midazolam in case of a trauma. ${ }^{13}$ However, nurses may decide to deviate from the protocol (stating reasons) based on the patient's condition and circumstances, or follow advices from regional protocols, e.g. using paracetamol. Care provided by EMS nurses can be complemented by team members of an HEMS. Four HEMS, which are available on a $24 / 7$ basis for the whole country, are equipped with a specially trained team including a HEMS physician (trauma surgeon or anaesthesiologist), a registered flight nurse, and a pilot. This team is capable of delivering hospital-level medical care and advanced pain management at the accident site, including the use of anaesthesia and certain analgesics. At the ED, a triage nurse assigns an urgency level to the patient's health problem, ${ }^{14}$ and may decide to give a patient certain pain medications before treatment by a physician. Care at the ED is provided by registered physicians and nurses who followed additional training in emergency medicine.

\section{Guideline}

The national evidence-based guideline was developed in 2010 by a multidisciplinary working group consisting of representatives from all relevant professionals working in emergency care; general practitioners, EMS nurses, HEMS physicians and nurses, physicians and nurses working in the ED, 
nurse practitioners, physician assistants, anaesthesiologists and physicians of surgical and orthopaedic traumatology departments. The guideline offers professionals recommendations and indicators, concerning pain assessment, initial (non-) pharmacological pain treatment, and the organisation of pain management in the chain of emergency care. The guideline has been distributed among these organisations, but has not yet been actively implemented nationwide.

The guideline recommends to register pain scores, initial (non-)pharmacological pain treatment and time of administration of medication in the medical records. The guideline suggests to use the verbal Numeric Rating Scale (NRS), a scale from zero to ten with zero as no pain and ten as unbearable pain, to assess pain score. Pain needs to be assessed at least three times; at arrival, after (non-) pharmacological intervention, and at the end of the medical visit. Non-pharmacological treatment of patients with fractures, contusions, and soft tissue injuries should be delivered according to the RICE\#-criteria - Rest, Ice, Compression and Elevation.

According to the guideline, initial pharmacological pain management should be given as indicated by algorithms, designed specifically for ambulance EMS, HEMS, GP(C) and ED (Figures 1 to 3). These algorithms consist of several routes concerning pain treatment for mild $(N R S<4)$, moderate to severe (NRS 4-7) and unbearable pain (NRS>7). In the guideline, paracetamol is the pharmacological treatment of first choice, if necessary with additional use of non-steroidal anti-inflammatory drugs (NSAIDs) or opioids. Fentanyl and Morphine can be given for severe to unbearable pain during emergency care. Administering Ketanest can be considered in case of severe or unbearable pain in combination with hypovolemia.

In order to measure pain management performance by professionals and organisations in emergency care, the recommendations in the guideline are translated into essential indicators (Table 1).

\section{Data collection and selection of patient files}

Patient files were handled anonymously - without patient identification, e.g. name or address. For each organisation, databases were preselected before a random sample for analysis was drawn. The preselection was based on the following inclusion criteria: patient in need of emergency care due to 
a recent trauma - less than 24 hours before admission - aged 18 or older, ABCD-stabile, and verbally responsive (Glasgow Coma Scale $\geq 14$ ) at least once during treatment. Due to the severity of trauma treated by the HEMS and the lower number of HEMS calls (on average 45 non-cancelled flights per month in the study period), the preselection of the HEMS included patients with a Glasgow Coma Scale $\geq 8$. This included also unresponsive patients, in contrast to the other organisations. Exclusion criteria were: patients that had drowning injuries, had attempted suicide, were victim of domestic violence, and visited ED for wound inspection or removal of stitches. After pre-selecting the electronic patient files, around 150 cases were randomly selected from each database.

In order to assess the electronic patient files for essential indicators, the quality indicators and recommendations from the guideline were translated into a measuring tool which was used to screen information in the patient files. The quality indicator 'treatment according to guideline algorithms' was assessed through a step by step analysis of the information per patient on pain as measured by the NRS, followed by the advised pharmacological treatment (dose not taken into account).

\section{Data analysis}

We used descriptive statistics (percentages, means and standard deviations (SDs)) to analyse the results. Data were analysed using SPSS 21 (SPSS Inc., Chicago, IL).

\section{RESULTS}

This study included a random sample of 1,066 files out of the 12,407 assessed electronic files of patients treated by the seven organisations in the emergency care, including 155 files of GPC, 176 of EMS, 238 of HEMS, and 497 of ED (Table 2). 


\section{Characteristics of patients}

Table 3 shows the characteristics of the 1,066 trauma patients, of whom most were men $(51.1 \%$ at EMS to $73.1 \%$ at HEMS). The age of the trauma patients ranged from 18 to 100 , and varied from a mean age of 41.9 (SD 17.2) in the GPC to 57.3 (SD 23.2) in the EMS. Most patients treated in the GPC had injuries due to sports, while patients treated by the (H)EMS or ED often had injuries that occurred at home or during leisure, or in traffic. Mechanism of injuries often included a fall or collision (data not shown). Most patients had injuries to their extremities (up to $72.1 \%$ in the GPC). Patients treated by the (H)EMS and ED often had injuries to head/neck (up to 29.4\%). The GPC often treated patients with superficial injury (24.5\%), while the (H)EMS and ED often treated patients with fractures (up to $52.7 \%$ ). In $1.3 \%$ (GPC) to $10.1 \%$ (ED) of the electronic patient files it was registered that patients were under the influence of alcohol and/or drugs. Most electronic patient files did not contain information about the mental state of patients (data not shown).

\section{Guideline adherence and current practice of pain management}

Adherence to the guideline is presented in Table 4 . Table 5 reports current pain management practice based on the chart review.

\section{Organisation of pain management}

According to the registration in patient files, one fifth of patients of the HEMS and ED received pain treatment within 10 minutes after the $A B C D$ assessment. For the GPC and the EMS this indicator could not be calculated due to missing time and pain score registrations. Registration of pain scores was lacking in the GPC, and varied from $15 \%$ in the EMS up to $52 \%$ in the ED. The registration of nonpharmacological pain treatment varied from $14 \%$ in the EMS up to $49 \%$ in the HEMS. Pharmacological pain treatment was registered in $13 \%$ in the GPC up to $48 \%$ in the (H)EMS. Finally, the registration of the time of administration of analgesia was not or poorly reported in the GPC 
(0\%), EMS (1\%) and HEMS (13\%). However, over one in four electronic files in the ED contained a time of administration of analgesia (27\%).

\section{Pain assessment}

The files of the GPC, and one HEMS indicated that a systematic pain score with the NRS was not used. In the EMS professionals registered the NRS in $15 \%$ of the patients, while for the ED in $25 \%$ and the other HEMS in $57 \%$ of the files a pain score was reported. If pain was assessed, it was mostly registered as assessed once during treatment. Indicators on the effectiveness of pain management (pain assessment after medication, and acceptable pain level after treatment) could not be calculated for the GPC, EMS and ED, due to the missing time registrations of the pain score and administration of pharmacological pain treatment. For the HEMS half of the patients (52\%) had an acceptable pain score registered after pharmacological interventions.

\section{(Non-)pharmacological treatment}

Non-pharmacological treatment of patients treated by the GPC often consisted of a bandage (57.4\%), while treatment at the ED (64.5\%) and HEMS (77.8\%) often consisted of immobilization by splint or cast (Table 5). Pharmacological pain treatment was administered and registered in accordance to the algorithms of the guideline in $1 \%$ (HEMS) up to $27 \%$ (ED) of the patients. Guideline adherence for the GPC could not be assessed, because the recommendations regarding pain management in the guideline are based on intensity of pain, and the GPs reported no pain scores in the files.

In general, only the registered pain treatment at the ED often included paracetamol (35.5\%) or a combination with paracetamol (49.6\%). Half of the patients with severe or unbearable pain in the ED received paracetamol (50\%); this was in contrast with the HEMS, where no or hardly any paracetamol was (registered to be) administered to patients in severe or unbearable pain. Due to the often life-threatening injuries, patients in the HEMS were mostly treated without paracetamol (51.3\%), and were provided with more stronger analgesia like Ketanest or anaesthesia. The files 
indicated that patients of all severities received paracetamol $(45.0 \%)$, NSAID $(40.0 \%)$ or a combination of medications including paracetamol (15.0\%) in the GPC, and Fentanyl in the EMS (36.9\%) and HEMS (47.0\%).

Overall, the chart review showed that the current registration of pain management mainly (but poorly) focused on the administration of analgesia and not on the pain assessment and evaluation, with a maximum compliance rate of $52 \%$.

\section{DISCUSSION}

Our study showed that (registration of) current pain management in trauma patients in the chain of emergency care varies widely between healthcare organisations, and is suboptimal. The assessment of pain scores using standardized tools was only registered in zero to $52 \%$ of the electronic patient files per organisation. Registration of (non-)pharmacological pain treatment was found in less than half of the files. According to the available information in the electronic patient files, pharmacological pain treatment was not in accordance with the guideline in $73 \%$ to $99 \%$ of the files. The time of administration of medication was missing in $73 \%$ to $100 \%$ of the patient files. Reassessment of pain following administration of pain medication was recorded in half of the patient files by the HEMS, but not in any of the patient files of the other organisations.

Although the Dutch guideline has been distributed among the organisations in emergency care, it has not yet been actively implemented nationwide. As a first step in the implementation of the guideline, this study assessed where current practice deviates from the guideline recommendations. It is important to note that any differences found are not interpreted as violations, but rather as issues for which the reasons need to be explored. Thus, a next step is to explore reasons for deviations from the guideline, which may depend on the circumstances, patient characteristics and 
injury severity. For example, the HEMS provides care to patients with often life-threatening injuries and unstable vital signs, and their advanced pain management often does not start with the administration of paracetamol and reaches beyond the algorithm for initial pain management in the guideline. Before deciding on an implementation strategy, barriers and facilitators for guideline adherence in the chain of emergency care need to be identified.

A limitation of this study was the use of chart review to assess current practice. The data registered by the emergency care organisations were not primarily gathered for research purposes, and not all organisations had the ability to register all relevant data on pain management in their electronic patient files, because their databases were not designed for this purpose. For example, the GPC had no pain scores registered in their electronic patient files. Guideline adherence for the GPC could therefore not be assessed, because the guideline recommendations regarding pain management are based on intensity of pain.

In addition, it is known that chart reviews are prone to underreporting. It is therefore highly plausible that missing registration on certain factors of pain assessment or pain treatment does not mean that these proceedings did not occur in practice; i.e. not performed or not registered. The lack of registration of information can have several meanings; assessment and treatment has not been done but should have been provided, has not been done and has not been provided with good reasons, was not needed at all, or has been done and is provided but not registered. This challenges the determination of guideline adherence. Overall, compliance with the guideline in practice, particularly with regard to registration of pain management, could be higher. Uniform registration of data by all organisations on the organisation of pain, pain assessment, and pain treatment, is key to accurately evaluate early pain management in the chain of emergency care.

With these limitations in mind, our study is to our knowledge the first to report on pain management in trauma patients in a comprehensive sample of organisations in the chain of emergency care. Most 
studies on pain management in trauma patients focused on one specific organisation in the emergency care setting.

The finding that (registration of) current practice of pain management in trauma patients in emergency care is suboptimal, is not new. Earlier retrospective document and prospective cohort studies among respectively 1,407 and 450 patient of the EMS and ED showed that in the ambulance only $30 \%$ of the patients receive pain medication, ${ }^{3}$ and in the ED this is even less $(19 \%)^{1}$, and $69 \%$ to $86 \%$ still report pain at discharge. ${ }^{1}$ Furthermore, a high prevalence of oligoanalgesia (40\%) was identified in adult trauma victims transported by the HEMS ${ }^{15}$. Although pain is the major complaint for patients seeking emergency care, ${ }^{16}$ research showed that only $20 \%$ of the patients with minor trauma actually received adequate pain management, while $40 \%$ of these patients expected to receive analgesia in the ED. ${ }^{17}$ In contrast, our study shows a much larger amount of patients for whom pain medication is registered at admission; GPC (15\%), EMS (48\%), HEMS (48\%), and ED (46\%). Although compliance with the guideline differs across groups of healthcare professionals, the maximum compliance rate with the indicators registered is $52 \%$. The deviations in current practice from the quality indicators of the guideline were however expected, as the guideline has not yet been implemented nationwide.

The pain guideline requires professionals in the chain of emergency care to collaborate and synchronize their pain management strategies. This might pose a problem as these professionals are used to work autonomously. Their differences in professional background, working culture, organisation and financial incentives may influence their guideline adherence. ${ }^{18,19}$ As with all guidelines, it is not self-evident that the guideline will implement itself. Therefore, the development of a tailored implementation strategy for the multidisciplinary pain guideline is necessary. This implementation strategy should be based on a thorough analysis of the specific setting of the chain of emergency care and the different organisations involved in pain management.

\section{Conclusions}


In conclusion, this study showed that (registration of) current pain management in trauma patients in the chain of emergency care varies widely between healthcare organisations, and deviates from the Dutch guideline recommendations. In order to improve pain management and evaluate its effectiveness, we recommend to improve pain registration in electronic patient files and to identify barriers and facilitators related to the implementation of the Dutch national guideline in all emergency care organisations.

\section{Acknowledgements}

We would like to thank all organisations in the emergency care that participated in this study, and especially the participants of the "Emergency Pain Study Group" for their support, which are: F. van Eenennaam, P. van Grunsven, W. Heutz, W. Breeman, D. den Hartog, N. Hoogerwerf, S. van Vliet, E. de Vaal, D. Pols, D. Kuster, M. Edwards, P. Rood, C. Doggen, L. Schoonhoven, S. Berben, A. Westmaas, A. Scholten. We would also like to acknowledge ZonMw, The Netherlands Organisation for Health Research and Development, for their funding to make this study possible (project number 171203004) 


\section{REFERENCES}

1. Berben SA, Meijs TH, van Dongen RT, van Vugt AB, Vloet LC, Mintjes-de Groot JJ, et al. Pain prevalence and pain relief in trauma patients in the Accident \& Emergency department. Injury. 2008;39: 578-85.

2. Liu M, Ferrante F. Overview of pain mechanisms and neuroanatomy. In: Rosenber $A D$, Grande CM, Bernstein RL, editors. Pain management and regional anesthesia in trauma. London: WB Saunders; 2000. p. 29-46.

3. Berben SA, Schoonhoven L, Meijs TH, van Vugt AB, van Grunsven PM. Prevalence and relief of pain in trauma patients in emergency medical services. Clin J Pain. 2011;27: 587-92.

4. Gramke HF, Marcus MA, Sommer M, van Kleef M. [Postoperative pain management: guidelines, organization and techniques]. Ned Tijdschr Geneeskd. 2009;153: 975-9.

5. Steegers MA, Wilder-Smith $\mathrm{OH}$. [Late chronic pain after surgery is prevented with good perioperative analgesics]. Ned Tijdschr Geneeskd. 2009;153: 562-6.

6. Berben SA, Kemps HH, van Grunsven PM, Mintjes-de Groot JA, van Dongen RT, Schoonhoven

L. [Guideline 'Pain management for trauma patients in the chain of emergency care']. Ned Tijdschr Geneeskd. 2011;155: A3100.

7. Lewis KS, Whipple JK, Michael KA, Quebbeman EJ. Effect of analgesic treatment on the physiological consequences of acute pain. Am J Hosp Pharm. 1994;51: 1539-54.

8. Dunwoody CJ, Krenzischek DA, Pasero C, Rathmell JP, Polomano RC. Assessment, physiological monitoring, and consequences of inadequately treated acute pain. Pain Manag Nurs. 2008;9: S11-21.

9. Netherlands Association for Emergency Nurses. Pain management in trauma patients in the chain of emergency care (in Dutch) 2011. Available from: http://www.cbo.nl/Downloads/1307/Richtlijn\%20Pijnbehandeling\%20bij\%20traumapatienten\%20in \%20de\%20spoedzorgketen.pdf. 
10. National Association of Emergency Medical Technicians. PHTLS: Basic and Advanced Prehospital Trauma Life Support. St. Louis: Mosby Elsevier; 2007.

11. Giesen P, Smits M, Huibers L, Grol R, Wensing M. Quality of after-hours primary care in the Netherlands: a narrative review. Ann Intern Med. 2011;155: 108-13.

12. van lerland $\mathrm{Y}$, van Veen $\mathrm{M}$, Huibers $\mathrm{L}$, Giesen $\mathrm{P}$, Moll $\mathrm{H}$. Validity of telephone and physical triage in emergency care: The Netherlands Triage System Family Practice. 2011: 334-41.

13. Dutch Ambulance Institute. National Protocols for Paramedics, version 7.2 (in Dutch). Zwolle: Dutch Ambulance Institute; 2011.

14. Huibers L, Thijssen W, Koetsenruijter J, Giesen P, Grol R, Wensing M. GP cooperative and emergency department: an exploration of patient flows. J Eval Clin Pract. 2013;19: 243-9.

15. Albrecht E, Taffe P, Yersin B, Schoettker P, Decosterd I, Hugli O. Undertreatment of acute pain (oligoanalgesia) and medical practice variation in prehospital analgesia of adult trauma patients: a 10 yr retrospective study. Br J Anaesth. 2013;110: 96-106.

16. Cordell WH, Keene KK, Giles BK, Jones JB, Jones JH, Brizendine EJ. The high prevalence of pain in emergency medical care. Am J Emerg Med. 2002;20: 165-9.

17. Whiteley J, Goodacre S. Patient expectations of minor injury care: a cross-sectional survey. Emerg Med J. 2013.

18. Berben SA, Meijs TH, van Grunsven PM, Schoonhoven L, van Achterberg T. Facilitators and barriers in pain management for trauma patients in the chain of emergency care. Injury. 2012;43: 1397-402.

19. Ebben RH, Vloet LC, Verhofstad MH, Meijer S, Mintjes-de Groot JA, van Achterberg T. Adherence to guidelines and protocols in the prehospital and emergency care setting: a systematic review. Scand J Trauma Resusc Emerg Med. 2013;21: 9.

20. Bevolkingsontwikkeling; regio per maand [Internet]. 2013 [cited 05.02.2014]. Available from: http://statline.cbs.nl/. 
Table 1 Indicators of guideline 'Pain management for trauma patients in the chain of emergency care'

Numerator / Denominator

\section{Organisation of pain management}

Pain treatment within 10 min after $A B C D$ assessment Registration of pain score

Registration of non-pharmacological pain treatment Registration of pharmacological pain treatment

Registration of time administration of medication

\section{Pain assessment}

Using Numeric Rating Score (NRS)

Using NRS after administration of analgesics

Acceptable pain level after treatment

Using NRS three times during treatment

\section{Pharmacological treatment}

Paracetamol for moderate to severe pain (NRS $\geq 4$ )

Treatment according to algorithms in guideline
Number of patients pain treatment started $\leq 10$ min after triage or intake / Patients with pain Number of patients with registered pain score / Total number of patients

Number of patients with registered non pharmacological pain treatment / Total number of patients Number of patients with registered pharmacological pain treatment / Total number of patients Number of patients with registered time of administration of medication / Total number of patients

Number of patients pain assessed with NRS / Total number of patients

Number of patients with pain score after pain medication / Patients with pain medication Number of patients acceptable pain NRS $<4$ after medication / Patients pain score after medication Number of patients pain assessed at least three times / Total number of patients

Number of patients with NRS 4-10 receiving paracetamol / Patients with NRS 4-10

Number of patients receiving treatment according to guideline flowcharts / Total number of patients 
Table 2 Pre-selection, inclusion and random sample per organisation

\begin{tabular}{|c|c|c|c|c|}
\hline Region $^{1}$ & Organisation & Period & Patient files & Sample \\
\hline Rural/suburban & ED - general & January - March 2012 & 8,741 & 195 \\
\hline \multirow[t]{4}{*}{ Suburban } & GPC & January - March 2012 & 1,224 & 155 \\
\hline & EMS & January - March 2012 & 807 & 176 \\
\hline & HEMS & October 2011 - March 2012 & 287 & 120 \\
\hline & ED - academic & January - March 2012 & 690 & 157 \\
\hline \multirow[t]{2}{*}{ Urban } & HEMS & January - March 2012 & 245 & 118 \\
\hline & ED - academic ${ }^{2}$ & January - March 2012 & 413 & 145 \\
\hline Total & & & 12,407 & 1,066 \\
\hline
\end{tabular}

${ }^{1}$ Defined by number of inhabitants per agglomeration: rural/suburban 159.000; suburban 168.000, urban 1.000.000. ${ }^{20}$

2 Selection of patients with primary transport by EMS to the ED. 
Table $\mathbf{3}$ Characteristics of trauma patients

\begin{tabular}{|c|c|c|c|c|}
\hline $\begin{array}{l}\text { Organisation } \\
\text { (N electronic patient files) }\end{array}$ & $\begin{array}{c}\text { GPC, } N(\%) \\
N=155\end{array}$ & $\begin{array}{c}\text { EMS, } N(\%) \\
N=176\end{array}$ & $\begin{array}{c}\text { HEMS, } N(\%) \\
N=238\end{array}$ & $\begin{array}{c}\text { ED, } N(\%) \\
N=497\end{array}$ \\
\hline \multicolumn{5}{|l|}{ Gender } \\
\hline Men & 96 (61.9) & $90(51.1)$ & $174(73.1)$ & $303(61.0)$ \\
\hline Women & $59(38.1)$ & $86(48.9)$ & $64(26.9)$ & $194(39.0)$ \\
\hline \multicolumn{5}{|l|}{ Age } \\
\hline Mean (SD) & $41.9(17.2)$ & $57.3(23.2)$ & $45.1(18.9)$ & $45.1(20.8)$ \\
\hline $18-19$ & $10(6.5)$ & $5(2.8)$ & $17(7.1)$ & $31(6.2)$ \\
\hline $20-39$ & $62(40.0)$ & $44(25.0)$ & 95 (39.9) & 209 (42.1) \\
\hline $40-59$ & $59(38.1)$ & $41(23.3)$ & $75(31.5)$ & $121(24.3)$ \\
\hline 60 or older & $24(15.5)$ & $86(48.8)$ & $51(21.4)$ & $136(27.4)$ \\
\hline \multicolumn{5}{|l|}{ Cause of injury } \\
\hline Home and leisure & $7(16.7)$ & $77(55.4)$ & $37(23.6)$ & $218(47.7)$ \\
\hline Traffic & 5 (11.9) & $41(29.5)$ & $95(60.5)$ & $103(22.5)$ \\
\hline Sports & $20(47.6)$ & $15(10.8)$ & $10(6.4)$ & $50(10.9)$ \\
\hline Work & $10(23.8)$ & $2(1.4)$ & $9(5.7)$ & $54(11.8)$ \\
\hline Violence & $0(0.0)$ & $4(2.9)$ & $6(3.8)$ & $32(7.0)$ \\
\hline Unknown/Not registered & 113 & 36 & 81 & 40 \\
\hline \multicolumn{5}{|l|}{ Location of injury } \\
\hline Head/neck & $10(6.8)$ & $41(20.1)$ & $130(29.4)$ & $100(18.6)$ \\
\hline Face & $24(16.3)$ & $6(2.9)$ & $63(14.3)$ & $61(11.3)$ \\
\hline Thorax & $7(4.8)$ & $17(8.3)$ & $71(16.1)$ & $27(5.0)$ \\
\hline Abdomen & $0(0.0)$ & $10(4.9)$ & $39(8.8)$ & $10(1.9)$ \\
\hline Spinal cord & $0(0.0)$ & $19(9.3)$ & $28(6.3)$ & $26(4.8)$ \\
\hline Extremities & $106(72.1)$ & $111(54.4)$ & $111(25.1)$ & $315(58.4)$ \\
\hline Unknown/Not registered & 8 & 11 & 21 & 52 \\
\hline \multicolumn{5}{|l|}{ Observed assumed injury } \\
\hline Fracture & $9(5.8)$ & $48(52.7)$ & $109(23.8)$ & $146(29.0)$ \\
\hline Dislocation/luxation & $21(13.5)$ & $20(22.0)$ & $7(1.5)$ & $39(7.8)$ \\
\hline Intracranial injury & $0(0.0)$ & $0(0.0)$ & $69(15.1)$ & $35(7.0)$ \\
\hline Internal injury ${ }^{1}$ & $3(1.9)$ & $0(0.0)$ & $31(6.8)$ & $17(3.4)$ \\
\hline Open wound & $16(10.3)$ & $9(9.9)$ & $26(5.7)$ & $53(10.5)$ \\
\hline Injury to blood vessel & $17(11.0)$ & $6(6.6)$ & $31(6.8)$ & $7(1.4)$ \\
\hline Superficial injury & $38(24.5)$ & $1(1.1)$ & $63(13.8)$ & $77(15.3)$ \\
\hline Contusion & $0(0.0)$ & $2(2.2)$ & $71(15.5)$ & $64(12.7)$ \\
\hline Other & $51(32.9)$ & $5(5.5)$ & $51(11.1)$ & 65 (12.9) \\
\hline Unknown/Not registered & 0 & 93 & 24 & 80 \\
\hline \multicolumn{5}{|l|}{ Under influence } \\
\hline Alcohol & $2(1.3)$ & $8(4.5)$ & $19(8.0)$ & $50(10.1)$ \\
\hline Drugs & $0(0.0)$ & $0(0.0)$ & $0(0.0)$ & $1(0.2)$ \\
\hline Alcohol and drugs & $0(0.0)$ & $1(0.6)$ & $1(0.4)$ & $5(1.0)$ \\
\hline Unknown/Not registered & 153 & 167 & 218 & 441 \\
\hline
\end{tabular}

${ }^{1}$ Internal injury of thorax, abdomen and/or pelvis. 
Table 4 Guideline adherence

\begin{tabular}{|c|c|c|c|c|}
\hline $\begin{array}{l}\text { Organisation } \\
\text { (N electronic patient files) }\end{array}$ & $\begin{array}{c}\text { GPC } \\
N=155\end{array}$ & $\begin{array}{c}\text { EMS } \\
\mathrm{N}=176\end{array}$ & $\begin{array}{c}\text { HEMS } \\
\mathrm{N}=238\end{array}$ & $\begin{array}{c}\text { ED } \\
\mathrm{N}=497\end{array}$ \\
\hline & $\mathrm{N}(\%)$ & $\mathrm{N}(\%)$ & $\mathrm{N}(\%)$ & $\mathrm{N}(\%)$ \\
\hline \multicolumn{5}{|l|}{ Organisation of pain treatment } \\
\hline Pain treatment within 10 min after $A B C D$ assessment ${ }^{1}$ & $*$ & $*$ & $10(18)$ & $23(20)$ \\
\hline Registration of pain score (any scoring instrument) & $0(0)$ & $26(15)$ & $68(29)$ & $259(52)$ \\
\hline Registration of non-pharmacological pain treatment & $47(30)$ & $24(14)$ & $117(49)$ & $50(46)$ \\
\hline Registration of pharmacological pain treatment & $20(13)$ & $84(48)$ & $115(48)$ & $228(46)$ \\
\hline Registration of time administration of medication & $0(0)$ & $2(1)$ & $31(13)$ & $132(27)$ \\
\hline \multicolumn{5}{|l|}{ Pain assessment } \\
\hline Using Numeric Rating Score (NRS) & $0(0)$ & $26(15)$ & $68(29)$ & $126(25)$ \\
\hline After administration of pain medication ${ }^{2}$ & $*$ & $*$ & $27(48)$ & $*$ \\
\hline Acceptable pain level after treatment ${ }^{3}$ & $*$ & * & $14(52)$ & $*$ \\
\hline At least three times during treatment & $*$ & $3(2)$ & $25(11)$ & $13(3)$ \\
\hline \multicolumn{5}{|l|}{ Pharmacological treatment } \\
\hline Paracetamol for moderate to severe pain $(\mathrm{NRS} \geq 4)^{4}$ & $*$ & $6(32)$ & $1(3)$ & $37(50)$ \\
\hline Treatment according to algorithms in guideline & * & $22(13)$ & $3(1)$ & $132(27)$ \\
\hline
\end{tabular}

Percentages are presented and calculated by the number divided by the total number of patients per organisation.

1 Divided by number of patients with pain (HEMS: 56, ED: 115).

2 Divided by number of patients with pain medication (HEMS: 56 ).

${ }^{3}$ Divided by number of patients with a pain score after medication (HEMS: 27).

${ }^{4}$ Divided by number of patients with NRS 4-10 (EMS: 19, HEMS: 39, ED: 74).

* Cannot be calculated due to missing, not registered or unknown data. 
Table 5 Current practice of pain management

\begin{tabular}{|c|c|c|c|c|}
\hline $\begin{array}{l}\text { Organisation } \\
\text { ( } \mathrm{N} \text { electronic patient files) }\end{array}$ & $\begin{array}{c}\mathrm{GPC}, N(\%) \\
\mathrm{N}=155\end{array}$ & $\begin{array}{c}\text { EMS, } N(\%) \\
\mathrm{N}=176\end{array}$ & $\begin{array}{c}\text { HEMS, } N(\%) \\
\mathrm{N}=238\end{array}$ & $\begin{array}{c}\mathrm{ED}, N(\%) \\
\mathrm{N}=497\end{array}$ \\
\hline \multicolumn{5}{|l|}{ Type of scale } \\
\hline NRS & $0(0.0)$ & $26(100)$ & $68(100)$ & $126(47.5)$ \\
\hline VRS & $0(0.0)$ & $0(0.0)$ & $0(0.0)$ & $117(44.2)$ \\
\hline NRS and VRS & $0(0.0)$ & $0(0.0)$ & $0(0.0)$ & $16(6.0)$ \\
\hline Other & $0(0.0)$ & $0(0.0)$ & $0(0.0)$ & $6(2.3)$ \\
\hline No/Unknown/Not registered ${ }^{1}$ & 155 & 150 & 170 & 232 \\
\hline \multicolumn{5}{|l|}{ Number of assessments } \\
\hline Once & $0(0.0)$ & $18(69.2)$ & $28(35.4)$ & $229(88.4)$ \\
\hline Twice & $0(0.0)$ & $5(19.2)$ & $26(32.9)$ & $17(6.6)$ \\
\hline 3 times & $0(0.0)$ & $3(11.5)$ & $25(31.6)$ & $13(5.0)$ \\
\hline Unknown/Not registered ${ }^{1}$ & 155 & 150 & 159 & 238 \\
\hline \multicolumn{5}{|l|}{ Non-pharmacological } \\
\hline No & $1(2.1)$ & $6(25.0)$ & $22(18.8)$ & $2(0.9)$ \\
\hline Immobilization & $0(0.0)$ & $10(41.7)$ & $91(77.8)$ & $149(64.5)$ \\
\hline Ice & $5(10.6)$ & $0(0.0)$ & $0(0.0)$ & $1(0.4)$ \\
\hline Compression & $8(17.0)$ & $0(0.0)$ & $0(0.0)$ & $35(15.2)$ \\
\hline Elevation & $1(2.1)$ & $1(4.2)$ & $0(0.0)$ & $11(4.8)$ \\
\hline Bandage & $27(57.4)$ & $7(29.2)$ & $2(1.7)$ & $18(7.8)$ \\
\hline Other & $5(10.6)$ & $0(0.0)$ & $2(1.7)$ & $15(6.5)$ \\
\hline Unknown/Not registered ${ }^{1}$ & 108 & 152 & 121 & 266 \\
\hline \multicolumn{5}{|l|}{ Pharmacological } \\
\hline Paracetamol & $9(45.0)$ & 15 (17.9) & $0(0.0)$ & $81(35.5)$ \\
\hline NSAID & $8(40.0)$ & $0(0.0)$ & $0(0.0)$ & $6(2.6)$ \\
\hline Fentanyl & $0(0.0)$ & 31 (36.9) & $54(47.0)$ & $13(5.7)$ \\
\hline Morfine & $0(0.0)$ & $2(2.4)$ & $0(0.0)$ & $9(3.9)$ \\
\hline Nitrous $50 \%$ / oxygen $50 \%$ & $0(0.0)$ & $3(3.6)$ & $0(0.0)$ & $0(0.0)$ \\
\hline Other & $0(0.0)$ & $6(7.1)$ & $0(0.0)$ & $0(0.0)$ \\
\hline Combination with paracetamol & $3(15.0)$ & $14(16.7)$ & $2(1.7)$ & $113(49.6)$ \\
\hline Combination without paracetamol & $0(0.0)$ & $13(15.5)$ & $59(51.3)^{*}$ & $6(2.6)$ \\
\hline Unknown/Not registered ${ }^{1}$ & 135 & 92 & 123 & 269 \\
\hline
\end{tabular}

${ }^{1}$ Missing information can have several meanings; assessment/treatment has not been done/provided unfairly, has not been done/provided with good reasons, was not needed at all, or has been done/provided but not registered.

* HEMS provides advanced pain treatment, while the guideline is limited to initial pain treatment. 
Figure 1 Algorithm for pharmacological pain management in ambulance EMS

Protocol for initial pharmacological pain management in adult, evaluable trauma patients in ambulance Emergency Medical Services

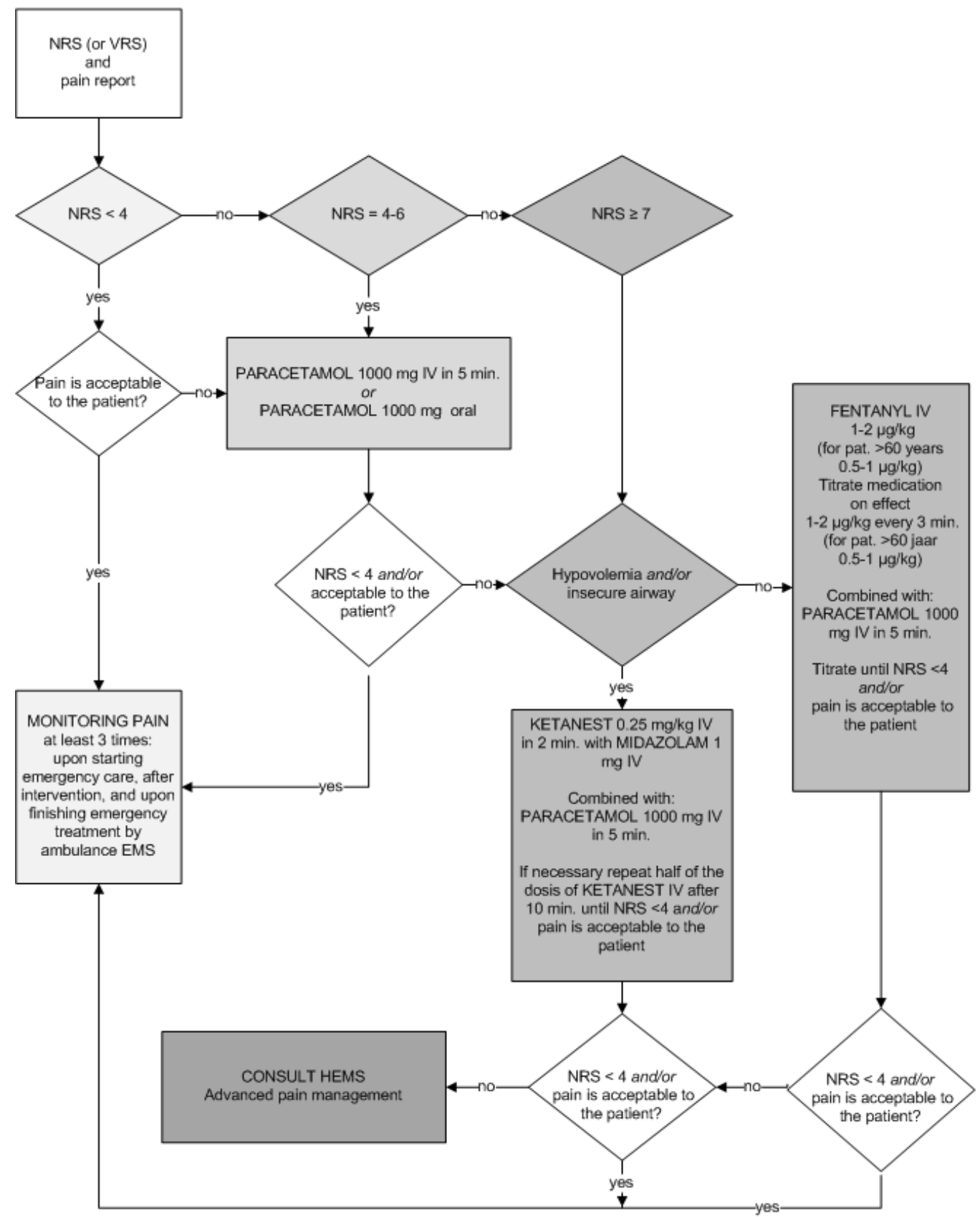


Figure 2 Algorithm for pharmacological pain management in HEMS

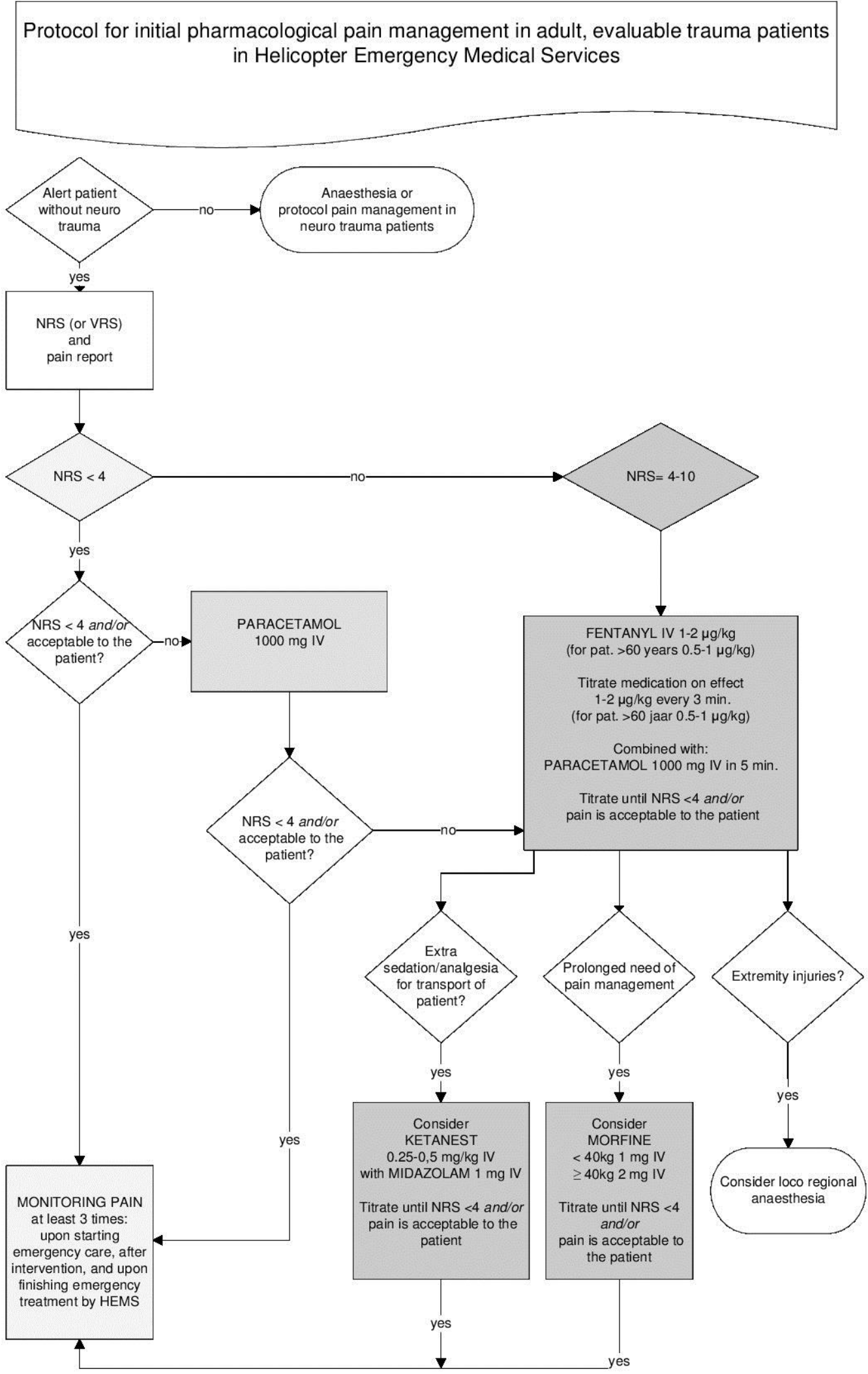


Figure 3 Algorithm for pharmacological pain management in $\mathrm{GP}(\mathrm{C})$ and $\mathrm{ED}$

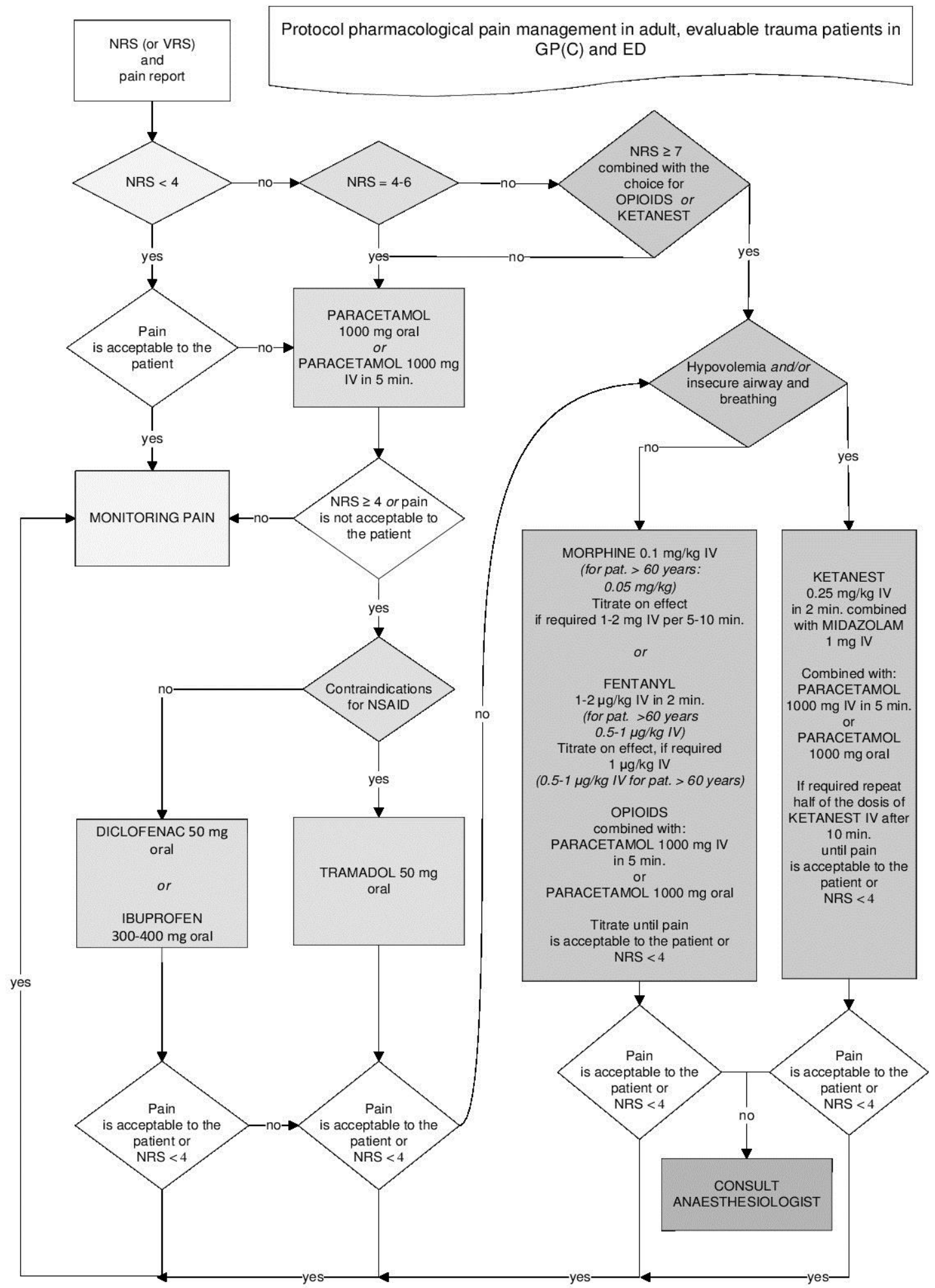

